This article was downloaded by: [The University of Manchester Library]

On: 03 July 2012, At: 04:19

Publisher: Psychology Press

Informa Ltd Registered in England and Wales Registered Number: 1072954 Registered office:

Mortimer House, 37-41 Mortimer Street, London W1T 3J H, UK

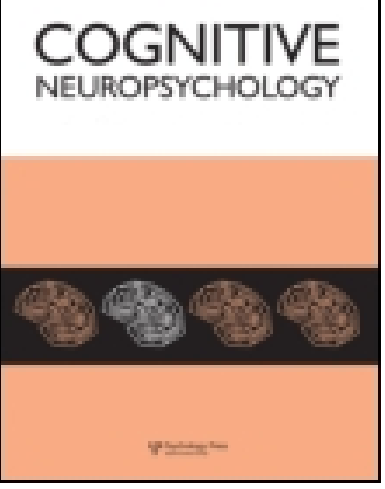

\title{
Cognitive Neuropsychology
}

Publication details, including instructions for authors and subscription information:

http:/ / www. tandfonline.com/loi/ pcgn20

\section{Finite case series or infinite single- case studies? Comments on "Case series investigations in cognitive neuropsychology" by Schwartz and Dell (2010)}

Matthew A. Lambon Ralph ${ }^{a}$, Karalyn Patterson ${ }^{b c} \&$ David C. Plaut ${ }^{d}$

${ }^{a}$ Neuroscience \& Aphasia Research Unit, School of Psychological Sciences, University of Manchester, Manchester, UK

${ }^{b}$ Department of Clinical Neurosciences, University of Cambridge, Cambridge, UK

${ }^{c}$ MRC Cognition \& Brain Sciences Unit, Cambridge, UK

${ }^{d}$ Department of Psychology and the Center for the Neural Basis of Cognition, Carnegie Mellon University, Pittsburgh, PA, USA

Version of record first published: 03 J ul 2012

To cite this article: Matthew A. Lambon Ralph, Karalyn Patterson \& David C. Plaut (2011): Finite case series or infinite single-case studies? Comments on "Case series investigations in cognitive neuropsychology" by Schwartz and Dell (2010), Cognitive Neuropsychology, 28:7, 466-474

To link to this article: http:// dx.doi.org/ 10.1080/ 02643294.2012.671765

\section{PLEASE SCROLL DOWN FOR ARTICLE}

Full terms and conditions of use: http://www.tandfonline.com/page/terms-and-conditions

This article may be used for research, teaching, and private study purposes. Any substantial or systematic reproduction, redistribution, reselling, loan, sub-licensing, systematic supply, or distribution in any form to anyone is expressly forbidden.

The publisher does not give any warranty express or implied or make any representation that the contents will be complete or accurate or up to date. The accuracy of any instructions, formulae, and drug doses should be independently verified with primary sources. The publisher shall not be liable for any loss, actions, claims, proceedings, demand, or costs or damages whatsoever or 
howsoever caused arising directly or indirectly in connection with or arising out of the use of this material. 


\title{
Finite case series or infinite single-case studies? Comments on "Case series investigations in cognitive neuropsychology" by Schwartz and Dell (2010)
}

\author{
Matthew A. Lambon Ralph ${ }^{1}$, Karalyn Patterson ${ }^{2,3}$ and David C. Plaut ${ }^{4}$ \\ ${ }^{1}$ Neuroscience \& Aphasia Research Unit, School of Psychological Sciences, University of Manchester, Manchester, UK \\ ${ }^{2}$ Department of Clinical Neurosciences, University of Cambridge, Cambridge, UK \\ ${ }^{3}$ MRC Cognition \& Brain Sciences Unit, Cambridge, UK \\ ${ }^{4}$ Department of Psychology and the Center for the Neural Basis of Cognition, Carnegie Mellon University, Pittsburgh, \\ PA, USA
}

\begin{abstract}
In this commentary, though acknowledging that a case-series approach in neuropsychology is not always possible, we set out a series of considerations that in our view make this approach generally superior to single-case study. We argue that case-series designs are crucial for theory-testing, assessment of computational models, evaluation of inter-patient variation (including selection criteria, patient homogeneity/heterogeneity, premorbid individual differences, etc.) and to establish solid foundations for the interpretation of behavioural dissociations and associations. We conclude by suggesting that, alongside other neuroscience techniques, case-series cognitive neuropsychology provides a crucial contribution to the future of clinical and cognitive neuroscience.
\end{abstract}

Keywords: Case series; Single-case studies; Individual differences; Variation; Dissociations; Associations.

As will be obvious from the responses to it in this issue of Cognitive Neuropsychology, the paper by Myrna Schwartz and Gary Dell (2010)—on the uses and abuses of case-series methodology in cognitive neuropsychology-has appropriately attracted a lot of interest. We endorse a great deal of the content of the Schwartz and Dell article (hereinafter, $\mathrm{S} \& \mathrm{D}$ ) and describe these sources of agreement below, although many have been noted in previous discussions of this topic (e.g., Lambon Ralph, Moriarty, \& Sage, 2002; Patterson \&
Plaut, 2009). We also have a few, mainly minor, questions or quibbles in connection with some of these nods of our heads; these are also addressed below. It seems appropriate, however, to start by nailing our colours to the mast.

All researchers presumably realize that there is no one correct or best methodology, and further that-even if there were-it would probably not be feasible to apply it to every research question. Thus we know that a case-series approach in neuropsychology is not always possible, and indeed all

Correspondence should be addressed to Matthew A. Lambon Ralph, Neuroscience \& Aphasia Research Unit, School of Psychological Sciences, University of Manchester, Manchester, UK. (E-mail: matt.lambon-ralph@manchester.ac.uk). 
three of us have performed single-case studies, either by choice or by necessity. If we have a significant disagreement with $\mathrm{S} \& \mathrm{D}$, it comes down to that phrase "by choice or by necessity". We interpret their target article as claiming either (a) that singlecase and case-series designs are equally valuable, and that the choice between them might be simply described as different horses for different courses, or else (b) that single-case studies remain the technique of choice in cognitive neuropsychology but that this preferred approach can be usefully complemented by case series. Although, of course, we agree that both methodologies can and do provide valuable information, we have a strong bias towards the case-series approach. We used to do single-case studies for two reasons: (a) like many researchers in cognitive neuropsychology, as nonclinicians we relied on neurologists or speech therapists to refer patients to us and were very grateful for even single cases to study; (b) we had not yet fully understood the value of case series. Nowadays, we would probably perform single-case studies for one reason only: that we could not find a second (or Nth) case like the one already identified.

We begin by dealing with the three principal points in the first main section of S\&D's article, "Introduction to the case series design".

First of all, in a contrast between case-series and single-case studies, $\mathrm{S} \& \mathrm{D}$ say that, in a case series "the cognitive analysis tends to be circumscribed. Typically, tests are . . . administered or reported only if they bear on . . . the study hypothesesthat is, there is not a systematic attempt to fully characterize the deficits of each individual" (p. 479). We agree partially with this point but view it as a positive rather than a negative feature of the case-series approach.

First, the reduction in breadth is often in reporting rather than in testing. That is, typically there are considerably more data regarding the patients in a case series than are reported in a given paper. The volume of data in this situation can often be too much to include in a reasonably sized, publishable paper. Beyond such publishing practicalities and of more scientific relevance, when the purpose of the study is to evaluate a bypothesis, some of the collected data are not directly relevant. For example, the article by Woollams, Lambon Ralph, Plaut, and Patterson (2007) reported reading data from 51 cases of semantic dementia. Results on aspects of the patients' abilities in various domains apart from reading (general cognitive status, perception, episodic memory, semantic memory) were (a) provided only for a limited set of tests, and (b) presented only as means and standard deviations for four subgroups of cases (mild, mild-moderate, moderatesevere, severe), not as individual-patient scores. With such a large $N$, a detailed characterization of each individual would certainly not have been feasible, but we also tend to doubt that anyone reading that paper would feel the need of it.

Furthermore, in some studies, the amount of relevant data may be so extensive that data reduction techniques can be utilized to aid in discovering underlying, meaningful patterns-something that is impossible with a single-case study. For example, a principal components analysis was used in this fashion in a study attempting to characterize homogeneity and heterogeneity in Alzheimer's disease (AD), with $N=55$ patients (Lambon Ralph, Patterson, Graham, Dawson, \& Hodges, 2003). In this specific instance, known clinical heterogeneity within the $\mathrm{AD}$ population is difficult to demonstrate formally using the test scores alone because it is masked by disease severity-which itself explains important features of the longitudinal profile in typical AD. Data reduction procedures take investigations a further step away from characterizing the profile of impaired and preserved abilities in individual patients, but once again, we consider this a gain rather than a loss. For example, in this specific investigation, individual patient scores were preserved and could be compared not only to control performance but also to patient profiles predicted solely on the basis of severity. Such studies, therefore, provide an example of how case-series analyses can augment the appreciation of individual patient data.

Secondly, S\&D argue that whereas single cases are selected on a cognitive basis, case-series studies often define the sample clinically, with all the trouble that may ensue, especially "unhelpful heterogeneity" (p. 479). This too is an important point to which we have two reactions. The first 
is that the presence of variation is a fact of life and thus an axiom of empirical science. As noted in our previous treatments of case-series methodology (Lambon Ralph et al., 2002; Patterson \& Plaut, 2009), variation has multiple sources, some of which are of no interest (e.g., measurement noise) whilst others can have important theoretical and clinical relevance (e.g., severity, individual differences - which, under the positive rubric of "personalized medicine", are considered to be the holy grail for contemporary clinical treatments). The crucial point here is that such sources of variation exist, and the selection of a datum does not negate this situation: It simply renders the single-case investigator silent on the issue because there is no basis to estimate the variation. In contrast, the case-series approach makes the variation apparent and, with its focus on preserving individual patient data, offers a basis for exploring it formally (the success of which will correspond to standard methodological factors such as sample size, stratification, sample selection, measurement reliability, and so on).

Our second reaction to this issue is that, in a sense, the single-case approach affords researchers the opportunity of not thinking very hard about how to select or define the disorder under investigation. Case-series investigations, on the other hand, force explicit consideration of the criteria for inclusion/exclusion, which may vary considerably from one study to another depending on the question(s) being asked. The basis for recruitment to a case series is not always/only a clinical diagnosis and may even be a specific pattern of data on a cognitive test. For example, the investigation of English past-tense morphological processing by Bird, Lambon Ralph, Seidenberg, McClelland, and Patterson (2003) started off with a large sample of patients $(N=50)$ defined clinically as having Broca's aphasia. This large cohort was then screened for a highly specific pattern of performance: greater success for irregular than regular verbs in past-tense tasks designed by Ullman et al. (1997). This screening reduced the selected number of study patients from 50 to 10 . There were two reasons for this selection criterion and emphasis on its resulting reduction in $N$. First, and most important, a performance advantage for irregular verbs was the pattern that Bird et al. were aiming to understand via further research, so it made sense to try to exclude the "unhelpful heterogeneity" of patients failing to show it. Secondly, the authors wanted other researchers to know that this pattern is by no means characteristic of all patients with a Broca-type aphasia, a point that had perhaps been somewhat glossed over in the Ullman et al. (1997) paper.

We completely endorse S\&D's point that caseseries investigations can provide evidence of both dissociations and associations, and indeed that both types of finding can emerge from both singlecase and case-series studies. S\&D note that Patterson and Plaut (2009) linked the case-series approach to a hunt for functional associations. $\mathrm{S} \& \mathrm{D}$ suggest that this is because the functional dissociations often prized in single-case studies support a highly modular view of cognition, whereas the functional associations predicted in many caseseries studies are more compatible with testing hypotheses arising from computational models (see below). This is true, but it was not the only reason for the link drawn by Patterson and Plaut. The other reason is a more factual, pragmatic point: Demonstration of a meaningful association in a single case is much harder, unless one component of the association also dissociated from other pertinent measures. For example, in category-specific semantic deficits, if a single patient's impaired knowledge of musical instruments associates with his deficit on living things and dissociates from his much better success rate on other man-made objects, this would constitute a publishable pattern (and was, in part, the basis of the differential feature-weighting hypothesis for category-specific deficits that arose from the seminal study of patient J.B.R.: Warrington \& Shallice, 1984). Otherwise, any single-case demonstration of an association, for example between semantic knowledge and reading of lower frequency irregular words, would be almost instantly dismissed, on the usual grounds that the brain regions responsible for two unrelated abilities just happen to be co-morbid in this patient. Given that a reliable association between semantic status and irregular-word reading reported for a large case series of patients with semantic 
dementia (Woollams et al., 2007) has been dismissed on these "accidental" grounds (Coltheart, 2010), it seems obvious that a single-case report would never get to first base. Our conclusion is that the case-series approach offers a range of outcomes, such as the identification of associations, dissociations, reliable individual differences, estimates of measurement noise, severity-based functions, and so on, many of which are not possible in single-case studies. Indeed, we would go further to suggest that the basis for linking a performance difference to an underlying cognitive-computational distinction or modular separation (i.e., what is classically considered to be a "dissociation") is more secure when demonstrated using a comparative case-series approach. ${ }^{1}$ This is because investigators can check that the between-group contrast is substantially different to the within-series patient variation and that it cannot be explained on the basis of measurement noise, severity, or other methodological considerations (various examples of comparative case-series studies can be found in the domain of semantic cognition: e.g., Jefferies \& Lambon Ralph, 2006; Lambon Ralph, Lowe, \& Rogers, 2007; Ogar et al., 2011).

It might be worth noting that, despite the emphasis on dissociations in traditional cognitive neuropsychology, it seems to us that functional associations, in some form, are a necessary feature of any reasonable theoretical account of cognition. Unless the theorist wants to propose a cognitive component that participates in only one task, there must surely always be a prediction of associations. For example, unlike the "triangle" approach to word reading (Dilkina, McClelland, \& Plaut, 2008; Plaut, McClelland, Seidenberg, \& Patterson, 1996; Seidenberg \& McClelland, 1989; Woollams et al., 2007), the dual-route computational (DRC) model of reading (Coltheart, 2010; Coltheart, Rastle, Perry, Langdon, \& Ziegler, 2001; Coltheart, Tree, \& Saunders 2010) argues for an orthographic input lexicon that can operate in isolation from word meaning. This position predicts that-all else being well-a patient should be able to read aloud irregular words (such as sew or pint in English) correctly in the face of degraded semantics for these wordsthat is, a dissociation between lexical and semantic processing. But the orthographic lexicon was, of course, not given to the human brain simply to prevent English-speaking patients with semantic deficits from making regularization errors in reading aloud. The presiding genius responsible for its existence knew that, one day, an experimental psychologist would invent the task of visual lexical decision, which in the DRC model also requires activation of an entry in the orthographic lexicon. So this model predicts, and correctly so, an association between irregular-word reading and lexical decision performance (Blazely, Coltheart, \& Casey, 2005), even while predicting a dissociation between irregular-word reading and semantic memory. In other words, both dissociations and associations will probably be observed on some level in any neuropsychological investigation, whether the participant cohort consists of a single case, a case series, or one or more groups, and regardless of the investigators' theoretical bent.

In addition to these three points in the introductory section of $\mathrm{S} \& \mathrm{D}$, there is one more, which occupies much less space. S\&D characterize case-series studies, at least in clinical medicine, as including no control groups. This may be true in medicine where normal function with little individual variation is a reasonable assumption for the non- or premorbid population. For many, if not most, cognitive measures, on the other hand, such an assumption is not secure; the great majority of the case-series studies that we know of in cognitive neuropsychology, therefore, have provided control data and would probably not have been accepted for publication without it.

The next section of the $\mathrm{S} \& \mathrm{D}$ article provides an admirable and extremely useful assessment of the value of case-series studies combined with quantitative models. They deal mainly with two topics:

\footnotetext{
${ }^{1}$ In a comparative case-series approach, two contrastive sets of patients are recruited, and each individual is investigated with the same test battery, forming two case series. Because the same assessments are used, this method allows the researcher to compare performance not only across individuals within a case series but also between the two groups.
} 
(a) the nature and proportions of error types in object naming by normal and aphasic individuals, as predicted and simulated within computational models developed by the authors of the target article and other colleagues (e.g. Dell, Schwartz, Martin, Saffran, \& Gagnon, 1997) and evaluated with detailed and extensive data from poststroke aphasia, again by the authors and colleagues (e.g. Schwartz, Dell, Martin, Gahl, \& Sobel, 2006); (b) the impact of semantic deterioration on putatively "nonsemantic" abilities such as reading words aloud, as predicted and simulated within computational models developed by us and our colleagues (e.g., Plaut et al., 1996) and evaluated with data from patients with semantic dementia, again studied by us and colleagues (e.g., Woollams et al., 2007). Too many nods of the head make for boring reading, but we have nothing other than praise for the way in which $S \& D$ have described these two programmes of research. They make it abundantly clear how, in these instances, the caseseries approach to empirical data linked with explicit computational models was not only valuable but, in fact, essential to the endeavours.

S\&D then extend their blessing of the caseseries approach to what they call anatomical case series. In the example used to illustrate this point, Schwartz et al. (2009) employed VLSM (voxel-based lesion-symptom mapping) to identify a highly specific left-temporal lobe region associated with a specific cognitive phenomenon-namely, the occurrence of semantic errors in object naming by patients with chronic poststroke aphasia. As S\&D note, cognitive neuropsychology-like cognitive psychology-mostly has not cared which part of the brain was achieving, or failing to achieve, the cognitive function of interest. If the necessary information was available, most publications in neuropsychology in the last quarter of the 20th century did indicate where the patient's lesion was, but the box-and-arrow models prevalent during that period typically had nothing to say about neuroanatomical localization of the boxes and arrows. S\&D's point is that, just as this neglect of the brain in "normal" cognitive psychology has been reversed by the availability of functional imaging, so widespread access to good structural (and sometimes functional) imaging of patients' brains has enabled a significant change in cognitive neuropsychology: It is now possible to do research in which the goal is not just to increase our cognitive understanding of a disorder like anomia but also to link specific features of it to specific brain regions. Not everyone considers this an important part of progress in the field of cognitive neuropsychology (Coltheart, 2010), but S\&D clearly do, and so do we (see Ueno, Saito, Rogers, \& Lambon Ralph, 2011, for a computational model of normal and impaired language function that explicitly sets out to localize its component functions in the human brain). Ironically, S\&D (p. 489) chose precisely the same passage from the same "guru" of cognitive psychology as we (Plaut \& Patterson, 2010) did in this regard: Shallice's (1988) claim that reliable information about lesion localization was not vital in neuropsychological research mainly because it was not feasible. At that time, almost 25 years ago, Shallice added that, in the future, "the situation might very well change" (1988, p. 214). Plaut and Patterson completed their article with the assessment that "the situation has changed" (2010, p. 14). S\&D clearly agree. One might wonder though whether these are more than parallel, independent activities; that is, cognitive theories are advanced through behavioural testing, and, independently, information about the associated lesions are accrued without this neuroanatomical information having any effect on cognitive theory. We would argue, instead, that it is possible for these and other neuroanatomical findings and hypotheses to be integrated into cognitive theories and computational models - and in doing so the resultant cognitive theory changes. Various examples can be found in the literature, and, in each case, the inclusion of anatomical information leads to an alternative or simpler cognitive explanation, and the models provide the basis for formally relating cognition and the brain (e.g., Lambon Ralph, McClelland, Patterson, Galton, \& Hodges, 2001; Plaut, 2002; Ueno et al., 2011).

S\&D then return to the problem of unhelpful heterogeneity and what to do about it. That is, if most but not all of the patients in a case series fit 
the predictions of the researchers' model, how do the researchers deal with the recalcitrant few? $\mathrm{S} \& \mathrm{D}$ describe two responses. One is essentially to treat the outliers as the basis for single-case studies, testing these particular cases on further measures that might explain why their performance deviates from what the model predicts. The example they give in detail is from a study reported by Fischer-Baum and Rapp (2012), who were able to demonstrate that one outlier case (in a total of 12) had an additional deficit that could account for the pattern of performance.

The second possible response, according to $\mathrm{S} \& D$, is essentially to redefine the inclusion criteria, thereby excluding the nonconforming cases on some specified grounds. This is actually more legitimate than it seems at first. S\&D give the good example of the set of patients in Dell et al. (1997), a study of aphasic naming errors. The goal was to understand errors of commission in naming, particularly semantic and nonword errors, but the original sample included some patients who made large numbers of omission errors. It turned out that these cases constituted most of the patients whose behaviour was poorly predicted by the computational model. Redefining the target population as "patients who make predominantly semantic and/or nonword errors in object naming" enabled a much better and more informative fit between model and data. This is an entirely reasonable move. Perhaps even more importantly, these two and other related examples (see below) demonstrate an important feature of the case-series approach-namely, that the limits of the theory or the hypotheses about unexpected data/patients are made open, transparent, and explicit for the rest of the community and thus can be explored by future investigations. Of course researchers would always like to be able to explain all of the data, but a model that accounts for a large proportion of results and fails only on a few should probably be developed further rather than abandoned. As one of our friends reminds us: The most successful model in historyquantum chromodynamics-cannot explain dark matter or dark energy, which together comprise the majority of stuff in the universe; yet physicists do not abandon the model, because it does so many other important things so well.

There is a third response to inhomogeneity that is not mentioned by S\&D but is one that we would encourage: to develop a theory that accounts not only for the central tendencies but also the variability in a population. While some of this variability is clearly a result of differences in the location and severity of brain damage, additional variability is no doubt due to various types of individual differences in cognitive and neural organization that predate the lesion. This issue is particularly challenging in cognitive neuropsychology as individuals become relevant for study only after suffering a neurological event that makes it impossible to determine their premorbid characteristics directly. Although individual differences are undeniably relevant to normal and impaired performance, consideration of them has been close to taboo in a cognitive neuropsychology based on single-case studies, but becomes feasible (if still challenging) if grounded in the study of case series.

This brings us to a specific point that we should address, although it is only lightly dealt with by S\&D: the suggestion by us and our colleagues that the outliers in the otherwise close relationship between semantic status and accuracy in reading lower frequency words with atypical spellingsound correspondences might be attributable to individual differences in the "division of labour" in reading (Woollams et al., 2007). As S\&D note but kindly do not dwell on, this essentially amounts to an unfulfilled promise within the first strategy mentioned above for dealing with heterogeneity. The theory proposes that there is variation within the normal population in the processing of words from orthography to phonology (O-to-P), specifically in the extent to which an interaction with word meaning enters the O-to-P computation. Suppose there are two individuals with semantic dementia: Case A who premorbidly relied heavily on semantics for reading aloud, and Case B who relied on semantics rather less. The prediction, not surprisingly, is that surface dyslexia will emerge earlier in the course of semantic deterioration for Patient $\mathrm{A}$ than for Patient B. This prediction was confirmed in the connectionist 
model of reading in Woollams et al. (2007), but it was only proposed, not confirmed, for the patient data. All 51 patients developed surface dyslexia during the longitudinal investigation, and most of the observations fell close to the regression function relating semantic status to success in reading lowfrequency exception words. There were, however, five exceptions to this good fit, defined as observations with standardized residuals \pm 2 : two patients (N.S. and J.P.) whose exception-word reading was significantly worse than that predicted by the regression function, and three (M.A., E.B., and M.G.) whose reading was too good to fit the function. We have already dealt extensively, in other publications, with the view held by some cognitive neuropsychologists that even one case with impaired semantic memory but relatively preserved exception word reading (even temporarily) should falsify the model - at least the version of the model without the factor of individual differences. Our point here is to acknowledge that, although we proposed that patients M.A., E.B., and M.G. would have had less than average reliance on semantics in premorbid reading aloud, we had no evidence to offer that this was indeed the case, as $\mathrm{S} \& \mathrm{D}$ comment. Until we are able to provide sufficient evidence of relevant individual variation in this regard, the individual differences hypothesis remains neither falsified nor unconditionally supported.

We do not discuss this issue any more here specifically with regard to surface dyslexia in semantic dementia because, as just noted, it has had substantial airing. We would, however, like to add one more point to S\&D's discussion of how to treat outliers, not so much statistically as theoretically. We interpret S\&D as saying, or at least meaning, that they share our view: That is, whereas even one black swan does destroy the theory that all swans are white, the performance of one or a few patients deviating from the predictions of a computational model need not destroy the model. It remains the case, however, that a number of researchers do think of testing cognitive neuropsychological models as equivalent to the swan colour test. A recent example is the paper by Tree and Kay (2006) presenting extensive data on a single case of phonological dyslexia.
J.H. did not show the association between poor nonword reading and deficits on nonreading phonological tasks that has been proposed as meaningful and demonstrated in several case-series studies (e.g., Crisp \& Lambon Ralph, 2006; Patterson \& Marcel, 1992; Rapcsak et al., 2009).

Tree and Kay's (2006) data certainly need to be taken seriously. All we want to say, and we think that $\mathrm{S} \& \mathrm{D}$ would agree, is that it would be easier to deal with these results if J.H. had been part of a case series rather than a single case. For example, across five different sets of nonwords, J.H.'s reading scores ranged from $58 \%$ to $84 \%$. Now take the case-series study by Crisp and Lambon Ralph (2006) arguing for an association between nonword reading and general phonological impairment. There is one test of nonword reading in common between the Tree and Kay study and the one by Crisp and Lambon Ralph: the 24-item nonword reading test from the PALPA (Psycholinguistic Assessments of Language Processing in Aphasia; Kay, Lesser, \& Coltheart, 1992). Patient J.H.'s score on this was $58 \%$. In the Crisp and Lambon Ralph study, 1 of the 12 patients also scored $58 \%$ on this test, and this was the top score: The rest of the cases had scores between $0 \%$ and $54 \%$, and 8 of the 12 scored less than 33\%. We are not disputing the claim that patient J.H. was a case of phonological dyslexia- $58 \%$ is an abnormal score, though he did better on other sets of nonwords. Our only point is that, had J.H. been embedded in a case series, there might be some hope of discovering whether his success on the same range of nonword-reading tests was consistently above average and, more importantly, how his performance on other kinds of assessments resembled or deviated from that of the other cases. As things stand, we have no clear way of deciding how black a swan he is.

This issue leads us on to a final point that we would like to make, which is in fact the basis for the title of this commentary on S\&D. It is a view that we attribute to our much-missed colleague and friend, Eleanor Saffran. Although much of Eleanor's extensive research output in cognitive neuropsychology consisted of singlecase studies, the approach worried her. She knew 
that, to make progress and have an impact, research must accumulate, and her fear was that an endless string of single-case studies might be incompatible with such accumulation. Back when Eleanor and author K.P. were first working in this field, there were relatively few researchers and relatively few (though indeed, often wellstudied) patients in the literature; when these researchers got together for discussion, they could simply call out a patient's initials: K.F.! W.L.P.! This is mildly reminiscent of the old joke about telling jokes by number, though of course a reference to such a patient was never met by laughter. The point is that it was met by recognition and knowledge. This is no longer true, and could not be true, given the explosion of work in this field of research. With the exception of a few especially famous cases like H.M. (who, ironically, was originally reported as a part of a case series: Scoville \& Milner, 1957), no one could be expected to keep in mind results from the myriad of single cases, nor even to read all of the publications of single cases on a topic like acalculia or phonological dyslexia or category-specific semantic impairment or whatever. And if one reads two different singlecase studies of phonological dyslexia that seem to offer discrepant findings (though as already noted, since the tests given and stimulus materials employed are rarely the same, it is often hard even to know whether the findings are genuinely different), then what? We share Eleanor's viewpoint: If cognitive neuropsychology is going to make progress, we need a finite number of case-series studies, not an infinite number of single cases.

\section{REFERENCES}

Bird, H., Lambon Ralph, M. A., Seidenberg, M. S., McClelland, J. L., \& Patterson, K. (2003). Deficits in phonology and past-tense morphology: What's the connection? Journal of Memory \& Language, 48, 502-526.

Blazely, A., Coltheart, M., \& Casey, B. J. (2005). Semantic impairment with and without surface dyslexia: Implications for models of reading. Cognitive Neuropsychology, 22, 695-717.
Coltheart, M. (2010). Lessons from cognitive science for cognitive neuropsychology: A reply to Patterson and Plaut (2009). Topics in Cognitive Science, 2, 3-11.

Coltheart, M., Rastle, K., Perry, C., Langdon, R., \& Ziegler, J. (2001). DRC: A dual route cascaded model of visual word recognition and reading aloud. Psychological Review, 108, 204-256.

Coltheart, M., Tree, J., \& Saunders, S. J. (2010). Computational modelling of reading in semantic dementia: Comment on Woollams, Lambon Ralph, Plaut and Patterson (2007). Psychological Review, 117, 256-272.

Crisp, J., \& Lambon Ralph, M. A. (2006). Unlocking the nature of the phonological-deep dyslexia continuum: The keys to reading aloud are in phonology and semantics. Journal of Cognitive Neuroscience, 18, 348-362.

Dell, G. S., Schwartz, M. F., Martin, N., Saffran, E. M., \& Gagnon, D. A. (1997). Lexical access in aphasic and nonaphasic speakers. Psychological Review, 104, 801-838.

Dilkina, K., McClelland, J. L., \& Plaut, D. C. (2008). A single-system account of semantic and lexical deficits in five semantic dementia patients. Cognitive Neuropsychology, 25, 136-164.

Fischer-Baum, S., \& Rapp, B. (2012). Underlying causes of letter perseveration errors in dysgraphia. Neuropsychologia, 50, 305-318.

Jefferies, E., \& Lambon Ralph, M. A. (2006). Semantic impairment in stroke aphasia vs semantic dementia: A case-series comparison. Brain, 129, 2132-2147.

Kay, J., Lesser, R., \& Coltheart, M. (1992). PALPA: Psycholinguistic Assessments of Language Processing in Aphasia. Hove, UK: Lawrence Erlbaum Associates.

Lambon Ralph, M. A., Lowe, C., \& Rogers, T. T. (2007). Neural basis of category-specific semantic deficits for living things: Evidence from semantic dementia, HSVE and a neural network model. Brain, 130, 1127-1137.

Lambon Ralph, M. A., McClelland, J., Patterson, K., Galton, C., \& Hodges, J. (2001). No right to speak? The relationship between object naming and semantic impairment: Neuropsychological evidence and a computational model. Journal of Cognitive Neuroscience, 13, 341-356.

Lambon Ralph, M. A., Moriarty, L., \& Sage, K. (2002). Anomia is simply a reflection of semantic and phonological impairments: Evidence from a case-series study. Aphasiology, 16, 56-82.

Lambon Ralph, M. A., Patterson, K., Graham, N. L., Dawson, K., \& Hodges, J. R. (2003). Homogeneity

COGNITIVE NEUROPSYCHOLOGY, 2011, 28 (7) 
and heterogeneity in mild cognitive impairment and Alzheimer's disease: A cross-sectional and longitudinal study of 55 cases. Brain, 126, 2350-2362.

Ogar, J. M., Baldo, J. V., Wilson, S. M., Brambati, S. M., Miller, B. L., Dronkers, N. F., et al. (2011). Semantic dementia and persisting Wernicke's aphasia: Linguistic and anatomical profiles. Brain and Language, 117, 28-33.

Patterson, K., \& Marcel, A. (1992). Phonological ALEXIA or PHONOLOGICAL alexia? In J. Alegria, D. Holender, J. Junca de Morais, \& M. Radeau (Eds.), Analytic approaches to human cognition. Amsterdam, The Netherlands: Elsevier.

Patterson, K., \& Plaut, D. (2009). "Shallow draughts intoxicate the brain": Lessons from cognitive science for cognitive neuropsychology. Topics in Cognitive Science, 1, 39-58.

Plaut, D. C. (2002). Graded modality-specific specialization in semantics: A computational account of optic aphasia. Cognitive Neuropsychology, 19, 603-639.

Plaut, D. C., McClelland, J. L., Seidenberg, M. S., \& Patterson, K. (1996). Understanding normal and impaired word reading: Computational principles in quasi-regular domains. Psychological Review, 103, 56-115.

Plaut, D., \& Patterson, K. (2010). Beyond functional architecture in cognitive neuropsychology: A reply to Coltheart. Topics in Cognitive Science, 2, 12-14.

Rapcsak, S. M., Beeson, P. M., Henry, M. L., Leyden, A., Kim, E., Rising, K., et al. (2009). Phonological dyslexia and dysgraphia: Cognitive mechanisms and neural substrates. Cortex, 45, 575-591.

Schwartz, M. F., \& Dell, G. S. (2010). Case series investigations in cognitive neuropsychology. Cognitive Neuropsychology, 27, 477-494.

Schwartz, M. F., Dell, G. S., Martin, N., Gahl, S., \& Sobel, P. (2006). A case series test of the interactive two-step model of lexical access: Evidence from picture naming. Journal of Memory and Language, 54, 228-264.

Schwartz, M. F., Kimberg, D. Y., Walker, G. M., Faseyitan, O., Brecher, A., Dell, G. S., et al. (2009). Anterior temporal involvement in semantic word retrieval: VLSM evidence from aphasia. Brain, 132, 3411-3427.

Scoville, W. B., \& Milner, B. (1957). Loss of recent memory after bilateral hippocampal lesions. Journal of Neurology, Neurosurgery, and Psychiatry, 20, 11-21.

Seidenberg, M. S., \& McClelland, J. L. (1989). A distributed, developmental model of word recognition and naming. Psychological Review, 96, 523-568.

Shallice, T. (1988). From neuropsychology to mental structure. Cambridge, UK: Cambridge University Press.

Tree, J. J., \& Kay, J. (2006). Phonological dyslexia and phonological impairment: An exception to the rule? Neuropsychologia, 44, 2861-2873.

Ueno, T., Saito, S., Rogers, T. T., \& Lambon Ralph, M. A. (2011). Lichtheim 2: Synthesising aphasia and the neural basis of language in a neurocomputational model of the dual dorsal-ventral language pathways. Neuron, 72, 385-396.

Ullman, M. T., Corkin, S., Coppola, M., Hickok, G., Growdon, J. H., Koroshetz, W. J., et al. (1997). A neural dissociation within language: Evidence that the mental dictionary is part of declarative memory, and that grammatical rules are processed by the procedural system. Journal of Cognitive Neuroscience, 9, 266-276.

Warrington, E. K., \& Shallice, T. (1984). Category specific semantic impairments. Brain, 107, 829-854.

Woollams, A. M., Lambon Ralph, M. A., Plaut, D., \& Patterson, K. (2007). SD-squared: On the association between semantic dementia and surface dyslexia. Psychological Review, 114, 316-339. 